Article

\title{
Overexpression of HMGA1 Figures as a Potential Prognostic Factor in Endometrioid Endometrial Carcinoma (EEC)
}

\author{
Antonio Palumbo Júnior ${ }^{1,2,+}$, Vanessa Paiva Leite de Sousa ${ }^{1,+}$, Francesco Esposito ${ }^{3}$ (D), \\ Marco De Martino ${ }^{3}$, Floriana Forzati ${ }^{3}$, Fábio Carvalho de Barros Moreira ${ }^{4}$, \\ Tatiana de Almeida Simão 1,5 (D), Luiz Eurico Nasciutti ${ }^{2}$, Alfredo Fusco ${ }^{1,3}$, \\ Luis Felipe Ribeiro Pinto ${ }^{1}$, Cláudia Bessa Pereira Chaves ${ }^{1,6}$ (D) and \\ Nathalia Meireles Da Costa $1, *$ (D)
}

1 Programa de Carcinogênese Molecular, Instituto Nacional de Câncer-INCA, Rua André Cavalcanti, 37 Centro, Rio de Janeiro, RJ 20231-050, Brazil; palumbo@icb.ufrj.br (A.P.J.); vpl_sousa@hotmail.com (V.P.L.d.S.); tasimao@gmail.com (T.d.A.S.); alfusco@unina.it (A.F.); lfrpinto@inca.gov.br (L.F.R.P.); claudia.bessa67@gmail.com (C.B.P.C.)

2 Laboratório de Interações Celulares, Instituto de Ciências Biomédicas, Universidade Federal do Rio de Janeiro Prédio de Ciências da Saúde—Cidade Universitária, Ilha do Fundão, A. Carlos Chagas, 373—bloco F, sala 26, Rio de Janeiro, RJ 21941-902, Brasil; luiz.nasciutti@histo.ufrj.br

3 Istituto di Endocrinologia e Oncologia Sperimentale-CNR c/o Dipartimento di Medicina Molecolare e Biotecnologie Mediche, Università degli Studi di Napoli “Federico II", via Pansini 5, 80131 Naples, Italy; francesco.esposito2@unina.it (F.E.); marco.demartino2@unina.it (M.D.M.); floriana.forzati@gmail.com (F.F.)

4 Divisão de Patologia, Instituto Nacional de Câncer-INCA, Rua Cordeiro da Graça, 156-Santo Cristo, Rio de Janeiro, RJ 20220-040, Brazil; fcbmoreira@hotmail.com

5 Laboratório de Toxicologia e Biologia Molecular, Departamento de Bioquímica, Instituto de Biologia Roberto Alcântara Gomes, Universidade do Estado do Rio de Janeiro, Av. 28 de setembro, 87 -fundos- $4^{\circ}$ andar, Rio de Janeiro, RJ 20551-030, Brazil

6 Seção de Ginecologia Oncológica, Hospital de Câncer II, Instituto Nacional de Câncer-INCA, Rua Equador, 835. Santo Cristo, Rio de Janeiro, RJ 20220-410, Brazil

* Correspondence: natmeireles@gmail.com; Tel.: +55-21-32076596; Fax: +55-21-32076536

+ These authors contributed equally to this work.

Received: 23 April 2019; Accepted: 6 May 2019; Published: 15 May 2019

check for updates

\begin{abstract}
Endometrioid endometrial carcinomas (EEC) are the most common malignant gynecologic tumors. Despite the increase in EEC molecular knowledge, the identification of new biomarkers involved in disease's development and/or progression would represent an improvement in its course. High-mobility group A protein (HMGA) family members are frequently overexpressed in a wide range of malignancies, correlating with a poor prognosis. Thus, the aim of this study was to analyze HMGA1 and HMGA2 expression pattern and their potential role as EEC biomarkers. HMGA1 and HMGA2 expression was initially evaluated in a series of 46 EEC tumors (stages IA to IV), and the findings were then validated in The Cancer Genome Atlas (TCGA) EEC cohort, comprising 381 EEC tumors (stages IA to IV). Our results reveal that HMGA1 and HMGA2 mRNA and protein are overexpressed in ECC, but only HMGA1 expression is associated with increased histological grade and tumor size. Moreover, HMGA1 but not HMGA2 overexpression was identified as a negative prognostic factor to EEC patients. Finally, a positive correlation between expression of HMGA1 pseudogenes-HMGA1-P6 and HMGA1-P7-and HMGA1 itself was detected, suggesting HMGA1 pseudogenes may play a role in HMGA1 expression regulation in EEC. Thus, these results indicate that HMGA1 overexpression possesses a potential role as a prognostic biomarker for EEC.
\end{abstract}

Keywords: endometrial carcinomas; cancer progression; molecular biomarkers 


\section{Introduction}

Endometrial carcinomas (ECs) are the most common malignant gynecologic tumors, with an estimated 320,000 new cases and 76,000 related deaths expected per year [1]. Etiological factors such as obesity, persistent anovulatory cycles, nulliparity and exogenous estrogen exposure are intrinsically associated with the malignancy. The main EC treatment is surgery, and adjuvant therapy can also be applied, depending on specific tumor behaviors, such as myometrial invasion [2-4]. Importantly, myometrial invasion rates increase along with tumor staging and represent an independent predictive outcome factor. Deep invasion is frequently associated with poor-differentiated tumors, lymph node metastasis, high recurrence rates and decreased overall survival [5,6]. Adenocarcinomas represent about $90 \%$ of EC cases and are subdivided in to type I or II according to both histological characteristics and clinical behavior [7]. Type I adenocarcinomas represent approximately $85 \%$ of cases and are known as endometrioid endometrial tumors (EEC). These tumors arise from previous hyperplasia, are estrogen-dependent, well- to moderately-differentiated, related to obesity and generally present good prognosis, with most of them being surgically curable [8]. Despite the increasing knowledge in molecular alterations present in EC, many questions remain unanswered. Enhanced effort in the identification of molecular markers involved in the development of the disease or its prognosis would represent an improvement in disease course [9].

The high-mobility group A proteins (HMGAs) are a family of small non-histone chromatin factors, encoded by the genes HMGA1 and HMGA2, which play a role in malignant cell transformation and progression of different tumors [10-17]. HMGAs proteins are involved in gene transcription regulation, acting through either the enhancement or suppression of transcription factors' activity by remodeling chromatin structure and orchestrating transcription factors multiprotein complexes recruitment $[18,19]$. While HMGA genes are expressed at very low levels in normal adult tissues, they are frequently overexpressed in a wide range of tumors, commonly predicting poor prognosis [20]. The mechanisms by which HMGA proteins are involved in cell transformation are mainly based on their capacity of modulating the expression of genes involved in cell proliferation and invasion control [20].

HMGA proteins have already been reported to be involved in development of female genital tract tumors. HMGA1-induced expression targeted to mice uterine tissues is capable of driving the development of uterine adenosarcoma [21]. Additionally, high levels of HMGA1 mRNA were detected in high-grade, aggressive uterine tumors when compared with less aggressive uterine neoplasms [22]. The involvement of HMGA2 in uterine neoplastic transformations is less clear. Nevertheless, increased levels of HMGA2 are only found in aggressive, invasive endometrium carcinomas [23].

Even though HMGA upregulation in tumors and its role in malignant cell transformation are well identified, their mRNA and protein levels regulation has not been comprehensively elucidated yet. Recent studies have demonstrated the involvement of epigenetic mechanisms, represented by non-coding RNAs, in both HMGA1 and HMGA2 regulation. For instance, two HMGA1 pseudogenes have been recently described, HMGA1P6 and HMGA1P7. These pseudogenes regulate HMGA1 protein levels by preventing their degradation mediated by miRNAs and possessing oncogenic characteristics $[24,25]$.

In order to assess whether HMGA1 and HMGA2 may serve as prognostic factors in EEC, we evaluated their mRNA and protein expression profile in EEC tumors ranging from stage IA to stage IV and correlated the clinicopathological features with the molecular findings. Additionally, we investigated HMGA1-P6 and HMGA1-P7 pseudogenes expression in order to verify whether they would play a role in $H M G A$ genes expression regulation.

\section{Material and Methods}

\subsection{Patients and Samples}

Forty-six patients with confirmed histological diagnosis of endometrioid endometrial carcinoma (EEC), who underwent surgical treatment between 2007 and 2009 at INCA (Brazilian National 
Cancer Institute, Rio de Janeiro, Brazil) and did not undergo chemo/radiotherapy, were included. Histological diagnosis was confirmed by two independent pathologists after surgical treatment. Six patients, who underwent total hysterectomy due to any clinical reason other than endometrial cancer in the same period, were also included (five atrophic and one proliferative endometrium samples). These patients, included in the study as controls for EEC development, did not undergo chemo/radiotherapy either and possessed clinicopathological characteristics similar/comparable to those of the EEC patients. Tumor size was measured by assessing the postoperative surgical specimen. Epidemiological and clinicopathological data were obtained through interviews by using a standardized questionnaire and from patients' medical records, respectively. Tumor and normal endometrial samples were formalin-fixed paraffin-embedded (FFPE). The protocol was approved by the Institutional Ethics Committee, and all patients signed a consent form.

\subsection{RNA Extraction, Reverse Transcription and RT-qPCR}

Total RNA was extracted from EEC and normal endometrial epithelium FFPE samples using PureLink ${ }^{\mathrm{TM}}$ FFPE Total RNA Isolation Kit (Invitrogen ${ }^{\circledR}$ ) according to manufacturer's instructions. All RNA samples were measured by spectrophotometry, and cDNA was synthesized from $1 \mu \mathrm{g}$ of RNA by using the SuperScript ${ }^{\mathrm{TM}}$ II Reverse Transcriptase (Invitrogen ${ }^{\circledR}$ ) protocol, and then real-time quantitative PCR (RT-qPCR) was performed by using SYBR Green Master Mix (Qiagen) and specific primers for the genes investigated. Primer sequences can be found on Table S1. Differential gene expression was calculated as described elsewhere [26].

\subsection{Immunohistochemistry}

Immunohistochemistry (IHC) was performed on $3 \mu \mathrm{m}$ paraffin sections of 20 EEC cases. HMGA1 (Abcam AB129153, working dilution 1:500) and HMGA2 (Abcam, AB52039, working dilution 1:50) immunostaining was performed as previously described $[27,28]$. The staining score evaluation was performed by a pathologist, blinded for clinicopathological parameters. For both proteins, scored cases were considered $1+$ when positive staining was present in up to $25 \%$ of tumor region, $2+$ when staining was present in $>26 \%$ and $\leq 50 \%$ of tumor region, $3+$ when present in $>51 \%$ and $\leq 75 \%$ of tumor region and $4+$ when $>76 \%$ of tumor region was positive. Anaplastic thyroid carcinoma samples were used as positive controls for HMGA1 and HMGA2 staining. As negative control, the primary antibody was replaced by the diluent solution.

\subsection{Analyses of HMGA1 and HMGA2 Expression Data Deposited in the Cancer Genome Atlas (TCGA)}

HMGA1 and HMGA2 expression data from 381 EEC samples and 20 endometrial histologically normal tumor-surrounding tissue as well as epidemiological and clinicopathological patients' data were downloaded from TCGA database.

\subsection{Statistical Analysis}

Frequencies of clinicopathological data and HMGA1 and HMGA2 mRNA expression levels were calculated. For continuous variables, descriptive analysis of central and dispersion tendencies was performed. To assess the relationship between mRNA expression levels and clinicopathological features, Fisher's exact, ANOVA or Kruskal-Wallis, and t-test or Mann-Whitney tests were used according to Gaussian distribution. For the correlation analyses we used Pearson's $r$ or Spearman's rho tests. The statistical analyses and ROC curve were performed with GraphPad Prism 5.0 (GraphPad Software Incorporated, San Diego, CA, USA). The final values were considered of statistical significance when $p<0.05$. Survival analyses were estimated by the Kaplan-Meier method and log-rank test, based on a confidence interval of $95 \%$. Data with $p<0.05$ were considered statistically significant. Variables with $p<0.2$ were selected for multivariate analysis. Finally, Cox regression was applied with the stepwise forward method. Survival analyses were performed by using R [29]. 


\subsection{Ethics Approval and Consent to Participate}

The use of the human samples was approved by the Ethics Committee of the Brazilian National Cancer Institute (INCA) (approbation number 091/2010, on the $28^{\text {th }}$ February 2011). All patients and healthy individuals, who kindly agreed to participate in the study, signed a consent form and authorized the scientific divulgation of the results obtained from the human samples donated.

\section{Results}

\subsection{Clinicopathological Features}

Table 1 shows EEC patients' clinicopathological characteristics. Patients' overall survival was $67.4 \%$ in a follow-up period of 108 months (median overall survival time of 66.7 months). Relapse was observed in $28.3 \%$ of the cases and, among them, $75.0 \%$ died of cancer. The median age of patients was 64 years, ranging from 42 to 83 years. Most of the patients presented family history of cancer (47.82\%), hypertension $(63.0 \%)$ and obesity or overweightness $(76.1 \%)$. The clinical characteristics of the six control women evaluated in the study are also illustrated in Table 1, showing that the only feature significantly different between cases and controls was their age. Association of overall survival and disease-free survival with all EEC patients' clinicopathological data was performed, and a significant association between tumor staging and overall survival ( $p=0.043 ; \mathrm{HR}=2.97)$ and disease-free survival $(p=0.006 ; \mathrm{HR}=6.93)$ was observed (Figure S1A,B).

Table 1. Clinicopathological characteristics of the 46 endometrioid endometrial carcinoma (EEC) patients and six controls in the study.

\begin{tabular}{|c|c|c|c|}
\hline Characteristics & Patients (\%) & Controls (\%) & $p$ \\
\hline Median age (years) & 64 & 39.5 & \multirow{2}{*}{0.003} \\
\hline Variation & $42-83$ & $35-72$ & \\
\hline \multicolumn{4}{|l|}{ Hypertension } \\
\hline Yes & $29(63.0)$ & $1(16.7)$ & \multirow{2}{*}{0.07} \\
\hline No & $17(37.0)$ & $5(83.3)$ & \\
\hline \multicolumn{4}{|l|}{ Diabetes } \\
\hline Yes & $9(19.6)$ & $0(0)$ & \multirow[t]{2}{*}{0.57} \\
\hline No & $37(80.4)$ & $6(100)$ & \\
\hline \multicolumn{4}{|l|}{ Heart diseases } \\
\hline Yes & $4(8.7)$ & $0(0)$ & \multirow[t]{2}{*}{1.00} \\
\hline No & $42(91.3)$ & $6(100)$ & \\
\hline \multicolumn{4}{|l|}{ Obesity or Overweight } \\
\hline Yes & $35(76.1)$ & $4(66.7)$ & \multirow[t]{2}{*}{0.63} \\
\hline No & $11(23.9)$ & $2(33.3)$ & \\
\hline \multicolumn{4}{|l|}{ Nulliparity } \\
\hline Yes & $6(13.0)$ & $0(0)$ & \multirow[t]{2}{*}{1.00} \\
\hline No & $36(78.3)$ & $6(100)$ & \\
\hline N/A & $4(8.7)$ & & \\
\hline \multicolumn{4}{|l|}{ Contraceptive use } \\
\hline Yes & $11(23.9)$ & $3(50)$ & \multirow[t]{2}{*}{0.33} \\
\hline No & $32(69.6)$ & $3(50)$ & \\
\hline N/A & $3(6.5)$ & - & \\
\hline \multicolumn{4}{|c|}{ Exogenous Estrogen Therapy } \\
\hline Yes & $0(0.0)$ & $1(16.7)$ & \multirow[t]{2}{*}{0.16} \\
\hline No & $42(91.3)$ & $5(83.3)$ & \\
\hline N/A & $4(8.7)$ & - & \\
\hline
\end{tabular}


Table 1. Cont.

\begin{tabular}{|c|c|c|c|}
\hline Characteristics & Patients $(\%)$ & Controls (\%) & $p$ \\
\hline \multicolumn{4}{|l|}{ Menopause } \\
\hline Yes & $38(82.6)$ & $3(50.0)$ & 0.06 \\
\hline No & $6(13.0)$ & $3(50.0)$ & \\
\hline N/A & $2(4.4)$ & - & \\
\hline \multicolumn{4}{|l|}{ FIGO Stage } \\
\hline IA & $10(21.7)$ & & \\
\hline IB & $10(21.7)$ & & \\
\hline II & $10(21.7)$ & & \\
\hline III & $10(21.7)$ & & \\
\hline IV & $6(13.0)$ & & \\
\hline \multicolumn{4}{|l|}{ Histological Grade } \\
\hline Well differentiated (G1) & $11(23.9)$ & & \\
\hline Moderate differentiated (G2) & $22(47.8)$ & & \\
\hline Poorly differentiated (G3) & $13(28.3)$ & & \\
\hline \multicolumn{4}{|l|}{ Lymphovascular Infiltration } \\
\hline Yes & $7(15.2)$ & & \\
\hline No & $29(63.0)$ & & \\
\hline $\mathrm{N} / \mathrm{A}$ & $10(21.8)$ & - & \\
\hline \multicolumn{4}{|l|}{ Myometrial Invasion } \\
\hline$<50 \%$ & $22(47.8)$ & & \\
\hline$\geq 50 \%$ & $24(52.2)$ & & \\
\hline \multicolumn{4}{|l|}{ Recurrence } \\
\hline Yes & $13(28.3)$ & & \\
\hline No & $33(71.7)$ & & \\
\hline
\end{tabular}

Fisher's exact test. N/A: Not informed.

3.2. HMGA1 is Overexpressed in EEC and Its Expression Positively Correlates with Increasing Tumor Staging, Grade and Size

First, we evaluated HMGA1 mRNA levels in 10 EEC samples from stage IA, 10 from stage IB, 10 from stage II, 10 from stage III, six samples from stage IV and in six normal, non-cancerous endometrial samples by RT-qPCR. There was a significant increase in the expression of HMGA1 in IB, II and III stage tumors, compared with normal endometrial samples. The increase in mRNA median expression values was of 1.3-, 2.2-, 2.3-, 2.7- and 2.1-fold in tumor Stage IA, IB, II, III and IV groups, respectively, when compared with control group (normal endometrium). A higher expression of HMGA1 mRNA levels in II and III tumor stages was also observed when comparing with IA stage tumor $(p=0.0001)$ (Figure 1A). Considering the histological grade of tumors, a statistically significant augmentation of HMGA1 expression was found in moderately and poorly differentiated tumors (G2 and G3, respectively) when compared with normal endometrial samples (Figure 1B). Furthermore, data analysis indicated a positive correlation between the increase of HMGA1 gene expression and tumors size $(\mathrm{r}=0.44, p=0.0028)$ (Figure 1C). A statistically significant association was also detected between HMGA1 expression levels and depth of myometrial invasion. Nevertheless, no significant association between HMGA1 gene expression and any other clinicopathological feature (Table S2) nor age $(\mathrm{r}=-0.09819, p=0.5162$, data not shown).

Next, aiming to verify whether HMGA1 protein expression follows the same pattern observed for mRNA levels, IHC was performed in the same six normal, non-cancerous endometrial tissues; five EEC samples from stage IA; five from stage IB; five from stage II; five from stage III and six samples from stage IV analyzed for gene expression. The 26 EEC samples assessed by immunohistochemistry were chosen randomly out of the total samples investigated for mRNA expression. As observed for gene expression, all samples were positive for HMGA1 staining and its intensity was higher in EEC samples from stages IB, II, III and IV when compared with samples from stage IA and normal endometrial 
samples (Figure 1D,I,J and Table S3). In addition, a significant positive correlation between gene and protein expression was observed $(\mathrm{r}=0.548 ; p=0.0038)$ (Figure 1$)$.
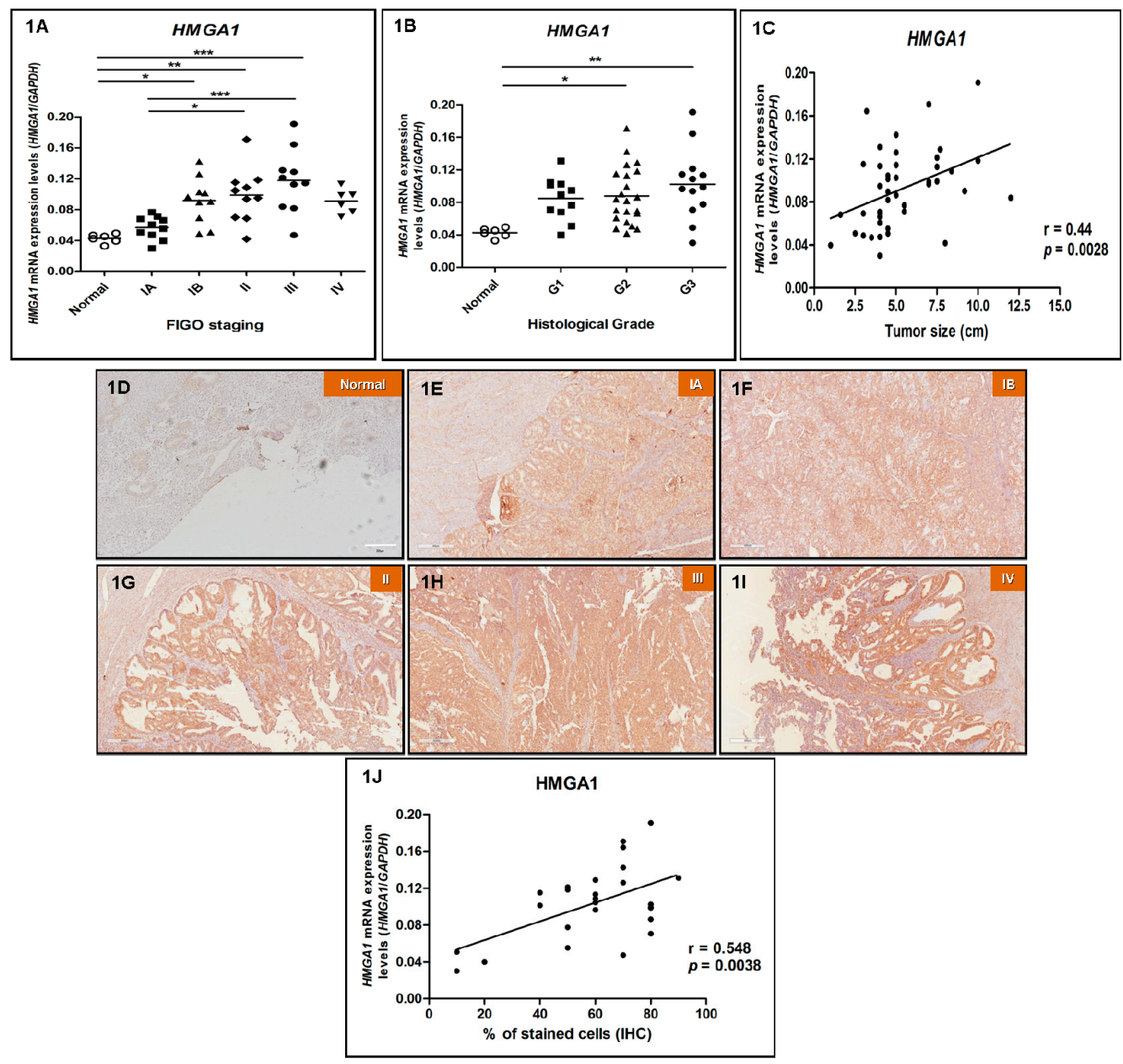

Figure 1. High-mobility group A protein 1 (HMGA1) mRNA and protein expression pattern in endometrioid endometrial adenocarcinomas (EEC). HMGA1 gene and protein expression analysis of the endometrioid endometrial adenocarcinoma (EEC) patients analyzed in this study, according to clinicopathological characteristics, by real-time quantitative PCR (RT-qPCR) and immunohistochemistry (IHC), respectively. (A) Tumor staging: HMGA1 mRNA expression performed in 10 EEC samples from stage IA, 10 from stage IB, 10 from stage II, 10 from stage III, six samples from stage IV and in six normal endometrial samples. (B) Histological grade: HMGA1 mRNA expression performed in 11 well differentiated (G1), 22 moderately differentiated (G2), 13 poorly differentiated (G3) EEC samples and in six normal endometrial samples. (C) Tumor size: Correlation between HMGA1 mRNA levels and tumor size. Evaluation of HMGA1 protein expression was performed in six normal endometrial tissue samples (D), five EEC samples from stage IA (E), five from stage IB (F), five from stage II (G), five from stage III (H) and five samples from stage IV (I) chosen randomly out of the 46 patients analyzed for gene expression. Magnification of photomicrographs: 100×. (J) Correlation between HMGA1 mRNA and protein expression. Statistical analyses were performed based on a confidence interval of $95 \%$. Data with $p<0.05$ were considered statistically significant. ${ }^{*}=p<0.05 ;{ }^{* *}=p<0.005 ;{ }^{* * *}=p<0.0005$.

\subsection{HMGA1P6 and HMGA1P7 Expression Follows the Same Pattern Observed for HMGA1 Expression}

Aiming to define whether HMGA1P6 and HMGA1P7 participate in HMGA1 expression regulation, their mRNA levels were investigated in the same EEC series (10 EEC samples from stage IA, 10 from stage IB, 10 from stage II, 10 from stage III, six samples from stage IV) and in six normal endometrial samples by RT-qPCR. A significant positive correlation was observed between HMGA1 and HMGA1P6 ( $\mathrm{r}=0.6070$; 
$p<0.0001$ ) (Figure 2A), HMGA1 and HMGA1P7 ( $\mathrm{r}=0.5383 ; p=0.0004)$ (Figure 2B) and HMGA1P6 versus HMGAP7 expression levels $(\mathrm{r}=0.9346 ; p<0.0001)$ (Figure $2 \mathrm{C}$ ). Additionally, a significant increase in HMGA1P6 and HMGA1P7 expression was observed along tumor staging augmentation. For HMGA1P6, a 2.45-, 15.09-, 5.72-, 7.98-, and 9.15-fold increase was detected in mRNA median expression values of tumor stages IA, IB, II, III and IV, respectively, when compared with normal endometrial samples' median expression value (Figure 2D). Similarly, for HMGA1P7, the augmentation in mRNA median expression values observed was of 3.19-, 9.79-, 8.82-, 10.94- and 6.28-fold in tumor stages IA, IB, II, III and IV, respectively, when compared with normal endometrial samples' median expression value (Figure 2E).

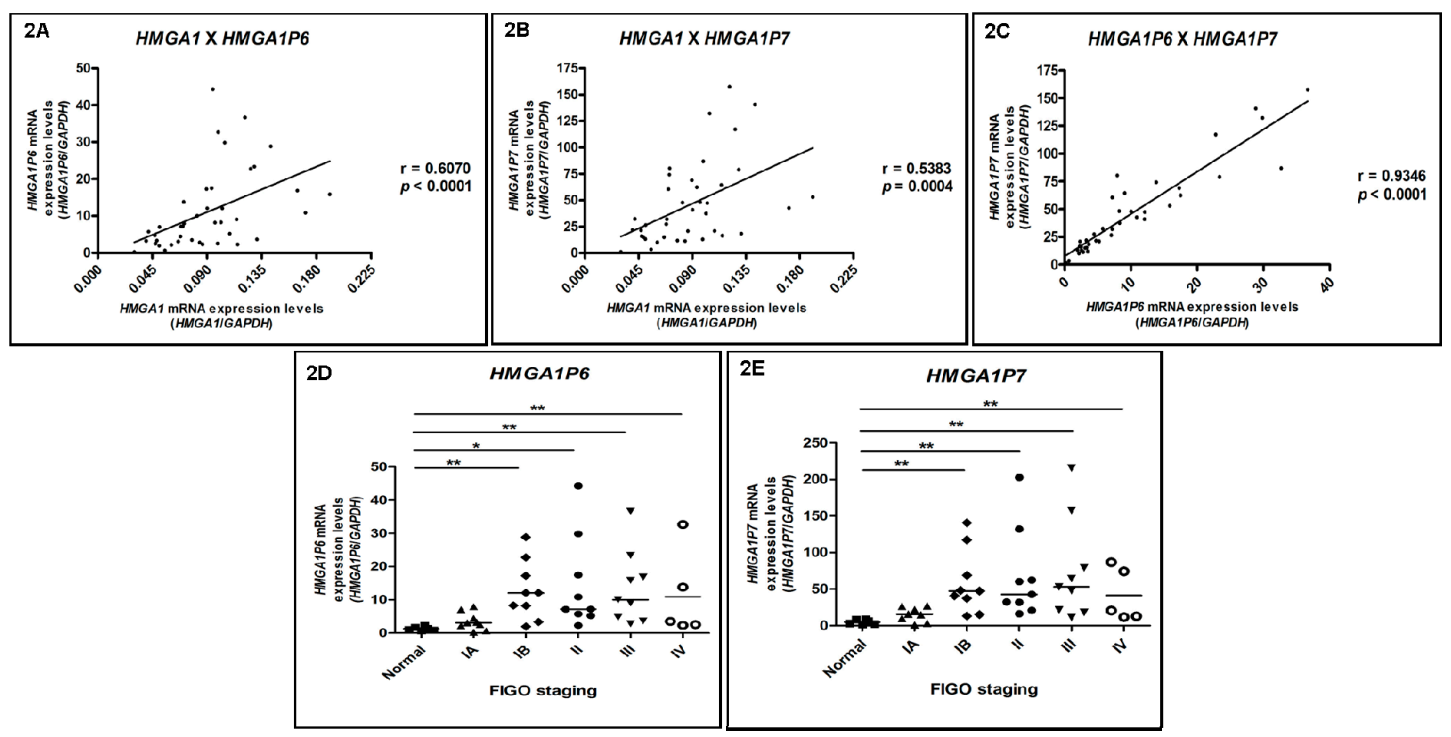

Figure 2. HMGA1-P6 and HMGA1-P7 mRNA expression pattern in endometrioid endometrial adenocarcinomas (EEC). Correlation of HMGA1 mRNA expression with those of HMGA1P6 and HMGA1P7 and evaluation of their expression, according to clinicopathological characteristics, by qRT-PCR. HMGA1P6 and HMGA1P7 gene expression was performed in six normal endometrial samples, 10 EEC samples from stage IA, 10 from stage IB, 10 from stage II, 10 from stage III and six samples from stage IV, and the (A) correlation between HMGA1 and HMGA1P6, (B) HMGA1 and HMGA1P7 and (C) HMGA1P6 and HMGA1P7 mRNA levels was evaluated. (D) HMGA1P6 and (E) HMGA1P7 mRNA expression distribution in the different tumor staging groups analyzed. Statistical analyses were performed based on a confidence interval of $95 \%$. Data with $p<0.05$ were considered statistically significant. ${ }^{*}=p<0.05$.

\subsection{HMGA2 Expression Does Not Correlate with Increasing Tumor Staging and Tumor Size in EEC}

In order to investigate $H M G A 2$ gene expression profile in EEC, its mRNA levels were assessed by RT-qPCR. The results shown in Figure 3A reveal that HMGA2 expression was positive in all 46 cases of EEC and normal tissue samples evaluated, and, although no statistically significance was observed between the different groups, HMGA2 mRNA median expression values of all different EEC stages evaluated were higher than that of normal endometrial samples. In particular, an evident increase in HMGA2 expression was observed in poorly differentiated tumors (G3) $(p=0.0268)$ when compared to normal endometrial samples (Figure 3B). However, differently from HMGA1 gene expression, there was no statistically significant association between $H M G A 2$ gene expression and tumor staging $(p=0.0654)$ (Figure 3A) nor tumor size (Figure 3C). Association analysis between HMGA2 expression data and patients' clinicopathological parameters was performed, and no significant association was detected (Table S4). HMGA2 expression levels were not associated with patients' age either $(\mathrm{r}=0.1512$, $p=0.3392$, data not shown). 

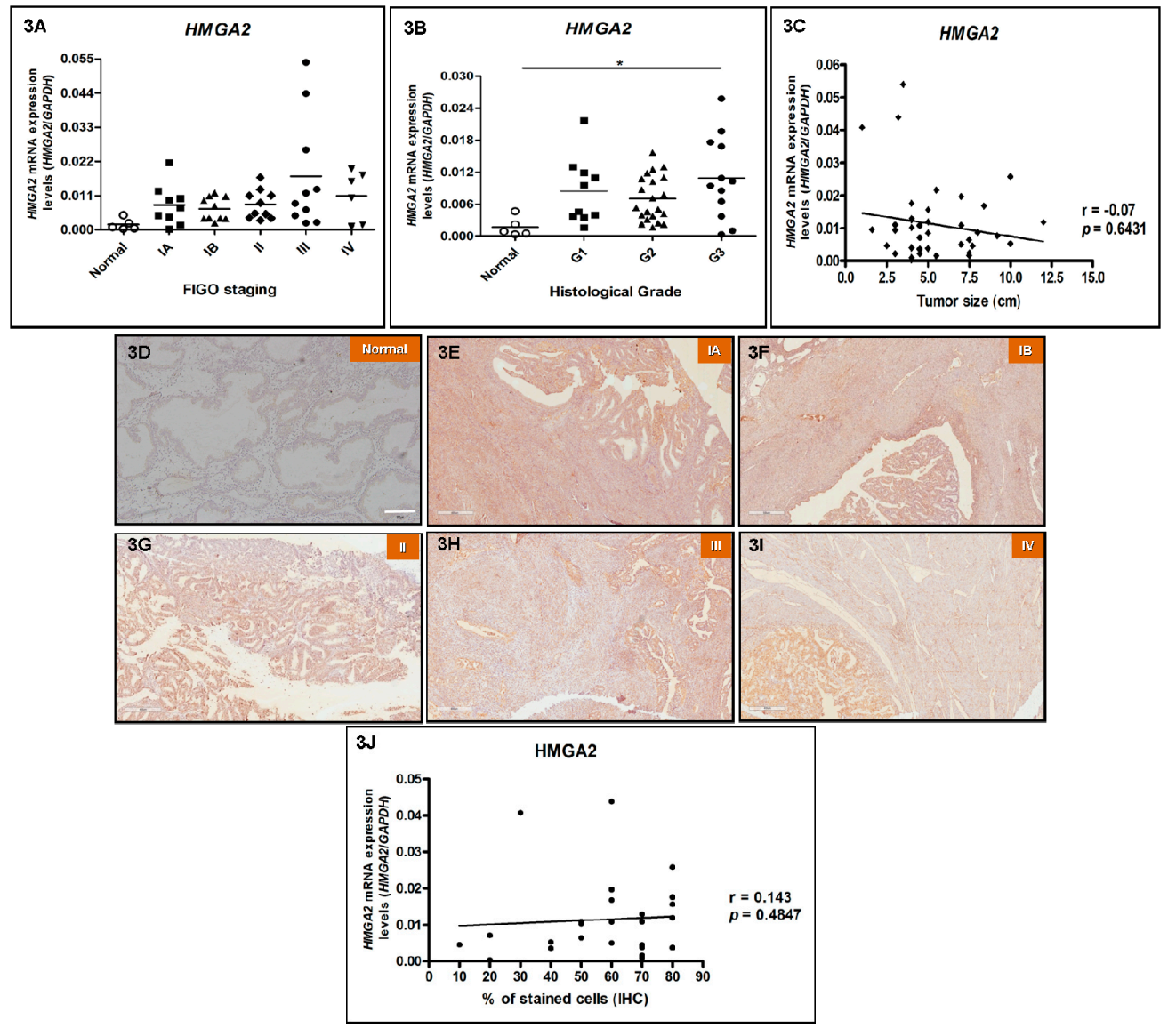

Figure 3. HMGA2 mRNA and protein expression pattern in endometrioid endometrial adenocarcinomas (EEC). HMGA2 gene expression analysis of the endometrioid endometrial adenocarcinoma (EEC) patients analyzed in this study, according to clinicopathological characteristics, by RT-qPCR and immunohistochemistry (IHC), respectively. (A) Tumor staging: HMGA2 mRNA expression performed in 10 EEC samples from stage IA, 10 from stage IB, 10 from stage II, 10 from stage III, six samples from stage IV and in six normal endometrial samples. (B) Histological grade: HMGA1 mRNA expression performed in 11 well differentiated (G1), 22 moderately differentiated (G2), 13 poorly differentiated (G3) EEC samples and in six normal endometrial samples. (C) Tumor size: Correlation between HMGA2 mRNA levels and tumor size. HMGA2 protein expression analysis in 25 endometrioid endometrial adenocarcinoma (EEC) patients by immunohistochemistry (IHC). Evaluation of HMGA2 protein expression was performed in six normal endometrial tissue samples (D), five EEC samples from stage IA (E), five from stage IB (F), five from stage II (G), five from stage III (H) and five samples from stage IV (I) chosen randomly out of the 46 patients analyzed for gene expression. Magnification of photomicrographs: 100×. (J) Correlation between HMGA2 mRNA and protein expression. Statistical analyses were performed based on a confidence interval of $95 \%$. Data with $p<0.05$ were considered statistically significant. ${ }^{*}=p<0.05$.

Regarding HMGA2 protein expression, IHC was performed in the same six normal endometrial tissues, five EEC samples from stage IA, five from stage IB, five from stage II, five from stage III and five samples from stage IV analyzed for gene expression in order to verify whether protein expression correlates with mRNA expression. The 26 EEC samples assessed by immunohistochemistry were chosen randomly out of the total samples investigated for mRNA expression. HMGA2 staining was not detected in normal endometrial samples, whereas all EEC samples were positive, nevertheless, the intensity of the staining was weak and no correlation between late tumor stages and increased intensity was observed (Figure 3D-I and Supplementary Table S5). Moreover, there was no significant correlation between HMGA2 gene and protein expression (Figure 3J). 


\subsection{Reanalysis of TCGA Data Confirms HMGA1 Upregulation in EEC and Reveals Its Impact on Patients' Survival}

Seeking to confirm the results achieved in a larger series, reanalysis of expression data from 381 EEC samples and 20 non-malignant tumor-adjacent mucosas available in TCGA database was performed. Among the 381 EEC samples investigated, 139 were classified as stage IA, 123 as stage IB, 35 as stage II, 70 as stage III, and 14 as stage IV. Regarding gene expression reanalysis of TCGA EEC cohort, similarly to the results obtained in our initial set of samples, HMGA1 was significantly overexpressed in tumors from all stages when compared with the non-cancerous tumor-adjacent endometrial tissues (Figure 4A). Considering the histological grade of tumors, a statistically significant augmentation of HMGA1 expression was found in well, moderately and poorly differentiated tumors (G1, G2 and G3, respectively) when compared with tumor-adjacent endometrial samples (Figure 4B). The increase of HMGA1 expression followed the loss of histological differentiation, since G3 EEC tumors presented significant higher HMGA1 mRNA levels when compared with G1 and G2 tumors (Figure 4B). Finally, since TCGA EEC cohort was large enough, survival analyses were performed, and they revealed a significant association between HMGA1 expression levels and overall survival $(p=0.03 ; \mathrm{HR}=1.93)$ in univariate analysis as well as with disease-free survival in both univariate $(p=0.00749 ; \mathrm{HR}=1.974)$ and multivariate analysis $(p=0.00334 ; \mathrm{HR}=1.7849)$, demonstrating its role as an independent prognostic factor (Figure $4 \mathrm{C}, \mathrm{D}$ and Table 2).

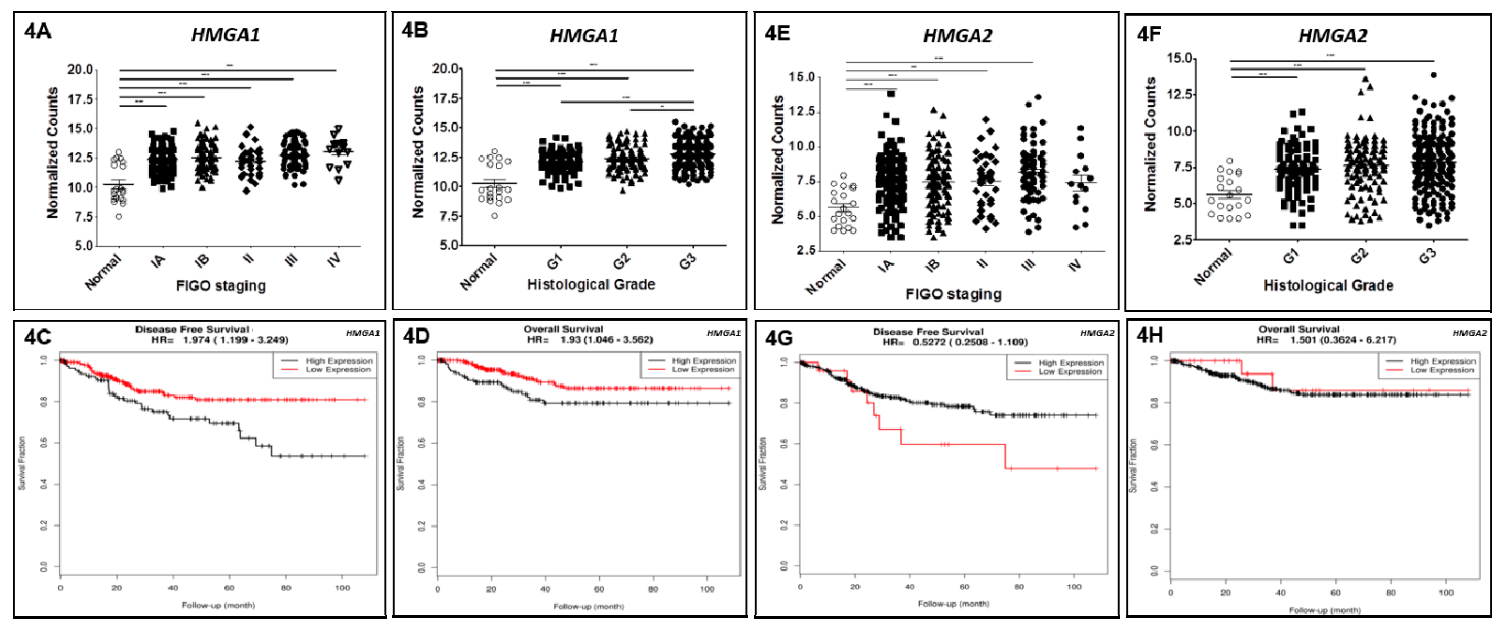

Figure 4. HMGA1 and HMGA2 expression in endometrioid endometrial adenocarcinomas (EEC) from The Cancer Genome Atlas (TCGA) cohort, and their potential as a prognostic biomarker. HMGA1 and HMGA2 gene expression reanalysis in EEC patients comprising TCGA cohort, according to clinicopathological characteristics. (A,E) Tumor staging: HMGA1 and HMGA2 expression was assessed in 139 EEC samples from stage IA, 123 from stage IB, 35 from stage II, 70 from stage III, 14 samples from stage IV and in 20 non-cancerous, tumor-adjacent endometrial tissue samples. (B,F) Histological grade: HMGA1 and HMGA2 expression reanalyzed in 95 well differentiated (G1), 107 moderately differentiated (G2), 179 poorly differentiated (G3) EEC samples and in 20 non-cancerous, tumor-adjacent endometrial tissue samples. $(\mathbf{C}, \mathbf{D}, \mathbf{G}, \mathbf{H})$ Survival analysis. (C,D). Evaluation of the association between the expression of HMGA1 on the 381 EEC patients' disease-free survival (C) $(\mathrm{HR}=1.974, \mathrm{CI}=1.199-3.249$, $p=0.00749)$ and overall survival (D) $(\mathrm{HR}=1.930, \mathrm{CI}=1.046-3.562, p=0.03)$ by univariate analysis. $(\mathbf{G}, \mathbf{H})$ Evaluation of the association between the expression of HMGA2 on the 381 EEC patients' disease-free survival $(\mathrm{G})(\mathrm{HR}=0.5272, \mathrm{CI}=0.2508-1.109, p=0.889)$ and overall survival $(\mathbf{H})(\mathrm{HR}=1.51$, $\mathrm{CI}=0.3624-6.217, p=0.67$ ) by univariate analysis. HMGA1 low expression < 12.92, HMGA1 high expression $\geq 12.92$; HMGA2 low expression $<7.68$, HMGA2 high expression $\geq 7.68$.

HMGA2 expression was also increased in TCGA EEC tumors from stages IA to III (Figure 4E) and from all histological grades (Figure 4F). Nevertheless, contrarily to HMGA1, there was no increment in $H M G A 2$ expression levels according to histological grade. Different from HMGA1 expression, survival 
analyses demonstrated that HMGA2 expression is not associated with either overall or disease-free survival of EEC patients in both univariate and multivariate analyses (Figure 4G,H and Table 2).

Table 2. Univariate and multivariate overall and disease-free survival analyses of the 381 endometrioid endometrial adenocarcinoma (EEC) patients from The Cancer Genome Atlas (TCGA) database evaluated in the study.

\begin{tabular}{|c|c|c|c|c|}
\hline Overall Survival & & & & \\
\hline & Category & HR & $95 \% \mathrm{CI}$ & $p$ Value \\
\hline \multicolumn{5}{|l|}{ Univariate Analysis } \\
\hline Age & $<$ median vs. $\geq$ median & 1.872 & $0.9818-3.571$ & 0.0569 \\
\hline Histological Grade & G3 vs. G2 vs. G1 & 2.381 & $1.457-3.892$ & 0.00054 \\
\hline FIGO Stage & Late vs. Early & 3.713 & $2.011-6.853$ & 0.0000274 \\
\hline HMGA1 & High vs. Low & 1.93 & $1.046-3.562$ & 0.03 \\
\hline HMGA2 & High vs. Low & 1.501 & $0.3624-6.217$ & 0.67 \\
\hline \multicolumn{5}{|l|}{ Multivariate Analysis } \\
\hline Age & $<$ median vs. $\geq$ median & 2.03 & $1.0417-3.940$ & 0.03749 \\
\hline Histological Grade & G3 vs. G2 vs. G1 & 2.013 & $1.2120-3.334$ & 0.00683 \\
\hline FIGO Stage & Late vs. Early & 3.33 & $1.7751-6.259$ & 0.00018 \\
\hline HMGA1 & High vs. Low & 1,315 & $0.7192-2.558$ & 0.34638 \\
\hline \multicolumn{5}{|l|}{ Disease-Free Survival } \\
\hline & Category & HR & $95 \% \mathrm{CI}$ & $p$ Value \\
\hline \multicolumn{5}{|l|}{ Univariate Analysis } \\
\hline Age & $<$ median vs. $\geq$ median & 1.628 & $0.9766-2.715$ & 0.0616 \\
\hline Histological Grade & G3 vs. G2 vs. G1 & 1,453 & $1.054-2.003$ & 0.0224 \\
\hline FIGO Stage & Late vs. Early & 1.922 & $1.128-3.275$ & 0.0163 \\
\hline HMGA1 & High vs. Low & 1.974 & $1.199-3.249$ & 0.00749 \\
\hline HMGA2 & High vs. Low & 0.5272 & $0.2508-1.109$ & 0.889 \\
\hline \multicolumn{5}{|l|}{ Multivariate Analysis } \\
\hline Age & $<$ median vs. $\geq$ median & 1.5992 & $0.9458-2.704$ & 0.0798 \\
\hline Histological Grade & G3 vs. G2 vs. G1 & 1.2960 & $0.9298-1.806$ & 0.1259 \\
\hline FIGO Stage & Late vs. Early & 1.8945 & $1.0702-3.196$ & 0.0276 \\
\hline HMGA1 & High vs. Low & 1.7849 & $1.0465-3.044$ & 0.0334 \\
\hline
\end{tabular}

Kaplan-Meier method and log-rank test. For multivariate analysis, Cox regression with the stepwise forward method. Multivariate analyses were adjusted by HMGA2 mRNA expression.

Next, in order to further investigate whether the gradual increase in HMGA1 expression along with tumor staging and its impact on EEC patients' survival could be related with a gain in tumor invasiveness, correlations analyses between HMGA1 and HMGA2 expression levels and those of the metalloproteinases MMP2 and MMP9 were performed using TCGA EEC expression data. A significant positive correlation was observed between $H M G A 1$ and MMP9 expression in late stages (III and IV) EEC tumors (rho $=0.38, p<0.01$ ), and between HMGA2 and MMP2 in early stages (I and II) tumors $($ rho $=0.21, p=0.008)$ (Table 3), suggesting a differential association pattern between the invasiveness markers and HMGA genes.

Table 3. Correlation analyses between HMGA genes and metalloproteinases ( $M M P$ ) expression assessed in samples from 381 endometrioid endometrial adenocarcinoma (EEC) patients from The Cancer Genome Atlas (TCGA) database evaluated in the study.

\begin{tabular}{cccccccccc}
\hline & \multicolumn{4}{c}{ HMGA1 } & \multicolumn{4}{c}{ HMGA2 } \\
& \multicolumn{1}{c}{ Early Stages } & \multicolumn{2}{c}{ Late Stages } & \multicolumn{2}{c}{ Early } & Stages & \multicolumn{2}{c}{ Late Stages } \\
\hline & rho & padj & rho & padj & rho & padj & rho & padj \\
MMP2 & 0.13 & 0.6 & 0.12 & 1 & $\mathbf{0 . 2 1}$ & $\mathbf{0 . 0 0 8}$ & 0.33 & 0.07 \\
MMP9 & 0.17 & 0.06 & $\mathbf{0 . 3 8}$ & $<\mathbf{0 . 0 1}$ & 0.12 & 0.8 & 0.003 & 1 \\
\hline
\end{tabular}




\section{Discussion}

In this study, we have analyzed the expression profile of HMGA genes in two different EEC samples series in order to access the potential of HMGA expression as a biomarker and, additionally, gain some insight on its tumor biology, since it is already known that alterations in HMGA expression are crucial for cancer development and progression [30].

Here, we report HMGA1 gene and protein overexpression in EEC samples from stage IA to IV, suggesting that HMGA1 overexpression may play a role in EEC development and progression. In fact, Tesfaye et al. showed that HMGA1 plays an important role in the genesis of uterine tumors, since the targeted expression of HMGA1 to the uterine tissue was able to drive the development of tumors which resemble the uterine adenosarcoma in a mouse model [21]. Additionally, the same study demonstrated that the up-regulation of the inflammatory mediator COX-2 represents the signaling mechanism through which HMGA1 induces uterine neoplastic transformation.

Our results also demonstrate a significant upregulation of HMGA1 expression following the increase in EEC histological tumor grade as well as a positive correlation between HMGA1 expression and tumor size and an association between HMGA1 levels and depth of myometrial invasion. Further, HMGA1 expression was revealed as an independent prognostic factor for EEC patients' disease-free survival. Thus, these results point out the role of HMGA1 expression as a prognostic marker for EEC patients and demonstrate that its upregulation is related to a poorer outcome. In line with these observations, Hillion and colleagues recently evaluated HMGA1 expression in three different histopathological types of uterine tumors (regardless of FIGO staging classification and histological grade within each of the groups investigated) and showed that HMGA1 is more expressed in high-grade uterine tumors, such as endometrial serous carcinoma and uterine carcinosarcoma, than in less aggressive ones, such as EEC, suggesting an increase in HMGA1 expression along with uterine tumor aggressiveness [22]. Additionally, reanalysis of TCGA EEC cohort performed in our study revealed a significant positive correlation between HMGA1 and MMP9 expression levels only in late stage samples. MMP9 is a metalloproteinase from gelatinase class, which is classically involved with degradation of elements harbored within the basal membrane, such as collagens, a biological event highly associated with tumor invasion [30]. The correlation between HMGA1 and MMP9 expression in late stage EEC samples raises the hypothesis that the upregulation of HMGA1 may trigger tumor invasiveness through MMP9 upregulation and consequently lead to patients' poorer prognosis. In fact, these data seem to be in accordance with previous studies that have shown the involvement of HMGA1 in the regulation of MMP9 expression along invasion and metastasis [31,32]. Finally, it has already been shown that HMGA1 overexpression is associated with patients' poor outcome in several human neoplasias, such as colon carcinomas [33,34], pancreatic adenocarcinomas [35,36], non-small cell lung cancer [37] and breast tumors [38].

Further, the expression analysis of HMGA1P6 and HMGA1P7 pseudogenes showed the same pattern observed for HMGA1 gene expression. Moreover, HMGA1 levels were positively correlated with those of HMGA1P6 and HMGA1P7, suggesting that these pseudogenes may participate in HMGA1 expression regulation. Indeed, it has been previously demonstrated that the overexpression of these pseudogenes leads to the increase in $H M G A 1$ levels by inhibiting driven suppression of HMGA1 synthesis by microRNAs acting as competitive endogenous RNA. Thus, upregulation of HMGA1P6 and HMGA1P7 increases HMGA1 protein expression, representing a mechanism of its regulation [24]. In agreement with our results, HMGA1P6 and HMGA1P7 expression was also upregulated in anaplastic thyroid and ovarian carcinomas and pituitary tumors, where it significantly correlates with HMGA1 overexpression [24,25].

Similar to the results observed for HMGA1 expression, HMGA2 was also detected as overexpressed in EEC tumors. Nevertheless, HMGA2 levels did not follow the increase in tumor histological grade, nor were they correlated with tumor size. Additionally, HMGA2 levels were not associated with EEC patients' prognosis. The involvement of $H M G A 2$ in endometrioid endometrial carcinogenesis is controversial. Romero-Pérez and colleagues reported that HMGA2 mRNA and protein overexpression participates in nonendometrioid carcinomas and endometrial carcinosarcomas development but not 
in that of EEC, suggesting its levels as a potential marker to distinguish between endometrioid and nonendometrioid endometrial tumors [39]. Accordingly, Wei and colleagues showed that HMGA2 is highly expressed in endometrium serous carcinomas (about $90 \%$ of the samples evaluated were positively stained for HMGA2), whereas it is absent in $60 \%$ of the EEC samples assessed. Additionally, among EEC samples expressing HMGA2, protein levels were barely detected when compared with endometrium serous carcinoma or with endometrium benign lesions, such as glandular dysplasia and intraepithelial neoplasia [23]. Controversially, a recent study published by Ma and colleagues [40] also reported the involvement of HMGA2 in endometrial cancer development and progression, demonstrating increased expression levels of HMGA2 in late stages, high-grade, and more invasive tumors. Further, in this study, HMGA2 overexpression was shown to be associated with poorer overall survival of patients in univariate analysis. However, in this study, tumors were evaluated collectively as endometrial carcinomas and not stratified by histological subtype, which represent a large variation in prognosis. Finally, Montserrat et al. demonstrated that HMGA2 is upregulated in EEC and that this phenomenon is associated with myometrial invasion [41]. HMGA2 upregulation and tissue invasion is a logical link, since HMGA2 is capable of regulating the epithelial-mesenchymal transition (EMT) process [42], and it could be one of the main mechanisms enabling invasion. However, although our results show HMGA2 upregulation in EEC samples, they may not account for tumor invasion as we did not observe any correlation between HMGA2 expression levels and those of the EMT regulators-SNAIL, SLUG and TWIST (data not shown). Additionally, there were no significant correlations between HMGA2 and gelatinases-MMP2 and MMP9—expression in late stage EEC samples. Therefore, more studies should be performed in order to further determine the role, if any, of HMGA2 in initiation and/or progression of endometrioid endometrial carcinogenesis.

HMGA1 and HMGA2 possess a highly similar structure and expression pattern. Nonetheless, studies using HMGA1 and HMGA2 null mice reported different phenotypes, suggesting that they have different functions [19]. In addition, a recent review proposed the differential induction of the HMGA proteins, depending on the cancer histological type, the induction of HMGA1 being an event usually observed in tumors originating from glandular tissues, whereas HMGA2 overexpression would participate in the development of tumors originating from epithelial cells [43]. This is in line with our findings showing that upregulation of HMGA1 but not of HMGA2 seems to participate in the initiation and/or progression of endometrioid endometrial carcinogenesis.

Together, these results point out HMGA1 overexpression as a potential prognostic biomarker for EEC.

Supplementary Materials: The following are available online at http://www.mdpi.com/2073-4425/10/5/372/s1, Figure S1: Survival analysis of the 46 endometrioid endometrial adenocarcinoma (EEC) patients analyzed in this study, according to tumor staging, Table S1: Sequences of the five pairs of oligonucleotides sequences used in the study, Table S2: Associations between HMGA1 gene expression and baseline characteristics of the 46 endometrioid endometrial adenocarcinoma (EEC) patients in the study, Table S3: HMGA1 protein expression by immunohistochemistry in 26 endometrioid endometrial carcinomas (EEC) patients in the study, Table S4: Associations between HMGA2 gene expression and baseline characteristics of the 46 endometrioid endometrial adenocarcinoma (EEC) patients in the study, Table S5: HMGA2 protein expression by immunohistochemistry in 26 endometrioid endometrial carcinomas (EEC) patients in the study.

Author Contributions: Conceptualization, A.P.J. and N.M.C.; Methodology, A.P.J., V.P.L.d.S., T.d.A.S., C.B.P.C., A.F., L.E.N., L.F.R.P. and N.M.D.C.; Formal analysis, A.P.J., V.P.L.d.S., M.D.M., F.E., F.F., T.d.A.S., F.C.B.M.; Investigation, A.P.J., V.P.L.d.S., A.F., L.F.R.P., C.B.P.C. and N.M.D.C.; Resources, L.E.N., A.F., L.F.R.P., C.B.P.C., N.M.D.C.; Data curation, V.P.L.d.S., C.B.P.C., N.M.D.C.; Writing—original draft preparation, A.P.J., V.P.L.d.S., N.M.D.C.; Writing—review and editing, L.E.N., A.F., L.F.R.P., C.B.P.C., N.M.D.C.; Project administration, N.M.D.C., L.F.R.P.; Funding acquisition, A.F., L.F.R.P.

Funding: This research was funded by Conselho Nacional de Desenvolvimento Científico e Tecnológico (CNPq-Brazil), Fundação de Amparo à Pesquisa Carlos Chagas Filho (FAPERJ—Brazil), Swiss Bridge Foundation and Associazione Italiana Ricerca sul Cancro (AIRC-Italy). Alfredo Fusco was granted with a Special Visiting Researcher (PVE) fellowship sponsored by CAPES.

Acknowledgments: We would like to thank Mariana Severo Ramundo and Pedro Nicolau Neto for the support with the survival analyses and for the scientific discussions.

Conflicts of Interest: The authors declare no conflict of interest. 


\section{References}

1. Siegel, R.L.; Miller, K.D.; Jemal, A. Cancer Statistics, 2018. CA Cancer J. Clin. 2018, 68, 7-30. [CrossRef] [PubMed]

2. Jaakkola, S.; Lyytinen, H.K.; Dyba, T.; Ylikorkala, O.; Pukkala, E. Endometrial cancer associated with various forms of postmenopausal hormone therapy: A case control study. Int. J. Cancer 2011, 128, 1644-1651. [CrossRef] [PubMed]

3. Dossus, L.; Rinaldi, S.; Becker, S.; Lukanova, A.; Tjonneland, A.; Olsen, A.; Stegger, J.; Overvad, K.; Chabbert-Buffet, N.; Jimenez-Corona, A.; et al. Obesity, inflammatory markers and endometrial cancer risk: A prospective case-control study. Endocr. Relat. Cancer 2010,17, 1007-1019. [CrossRef]

4. Morice, P.; Leary, A.; Creutzberg, C.; Abu-Rustum, N.; Darai, E. Endometrial Cancer. Lancet 2016, 387, 1094-1108. [CrossRef]

5. Uharcek, P. Prognostic factors in endometrial carcinoma. J. Obstet. Gynecol. Res. 2008, 34, 776-783. [CrossRef]

6. Dizon, D.S. Treatment options for advanced endometrial carcinoma. Gynecol. Oncol. 2010, 117, $373-381$. [CrossRef]

7. Bokhman, J.V. Two pathogenetic types of endometrial carcinoma. Gynecol. Oncol. 1983, 15, 10-17. [CrossRef]

8. Felix, A.S.; Yang, H.P.; Bell, D.W.; Sherman, M.E. Epidemiology of Endometrial Carcinoma: Etiologic importance of hormonal and metabolic influences. Adv. Exp. Med. Biol. 2017, 943, 3-46. [CrossRef]

9. Silva, J.L.; Paulino, E.; Dias, M.F.; Melo, A.C. Endometrial cancer: Redefining the molecular-targeted approach. Cancer Chemother. Pharmacol. 2015, 76, 1-11. [CrossRef]

10. Sgarra, R.; Pegoraro, S.; Ros, G.; Penzo, C.; Chiefari, E.; Foti, D.; Brunetti, A.; Manfioletti, G. High Mobility Group A (HMGA) proteins: Molecular instigators of breast cancer onset and progression. Biochim. Biophys. Acta Rev. Cancer 2018, 1869, 216-229. [CrossRef] [PubMed]

11. Resar, L.M. The high mobility group A1 gene: Transforming inflammatory signals into cancer? Cancer Res. 2010, 70, 436-439. [CrossRef] [PubMed]

12. Chou, B.K.; Mali, P.; Huang, X.; Ye, Z.; Dowey, S.N.; Resar, L.M.S.; Zou, C.; Zhang, Y.A.; Tong, J.; Cheng, L. Efficient human iPS cell derivation by a non-integrating plasmid from blood cells with unique epigenetic and gene expression signatures. Cell Res. 2011, 21, 518-529. [CrossRef]

13. Karp, J.E.; Smith, B.D.; Resar, L.S.; Greer, J.M.; Blackford, A.; Zhao, M.; Moton-Nelson, D.; Alino, K.; Levis, M.J.; Gore, S.D.; et al. Phase 1 and pharmacokinetic study of bolus-infusion flavopiridol followed by cytosine arabinoside and mitoxantrone for acute leukemias. Blood 2011, 117, 3302-3310. [CrossRef] [PubMed]

14. Nelson, D.M.; Joseph, B.; Hillion, J.; Segal, J.; Karp, J.E.; Resar, L.M. Flavopiridol induces BCL-2 expression and represses oncogenic transcription factors in leukemic blasts from adults with refractory acute myeloid leukemia. Leuk. Lymphoma 2011, 52, 1999-2006. [CrossRef]

15. Schuldenfrei, A.; Belton, A.; Kowalski, J.; Talbot, C.C., Jr.; Di Cello, F.; Poh, W.; Tsai, H.L.; Shah, S.N.; Huso, T.H.; Huso, D.L.; et al. HMGA1 drives stem cell, inflammatory pathway, and cell cycle progression genes during lymphoid tumorigenesis. BMC Genomics 2011, 12, 549-565. [CrossRef] [PubMed]

16. Belton, A.; Gabrovsky, A.; Bae, Y.K.; Reeves, R.; Iacobuzio-Donahue, C.; Huso, D.L.; Resar, L.M.S. HMGA1 induces intestinal polyposis in transgenic mice and drives tumor progression and stem cell properties in colon cancer cells. PLoS ONE 2012, 7, e30034. [CrossRef] [PubMed]

17. Shah, S.N.; Resar, L.M. High mobility group A1 and cancer: Potential biomarker and therapeutic target. Histol. Histopathol. 2012, 27, 567-579. [CrossRef] [PubMed]

18. Sumter, T.F.; Xian, L.; Huso, M.; Koo, M.; Chang, Y.T.; Almasri, T.N.; Chia, L.; Inglis, C.; Reid, D.; Resar, L.M. The high mobility group A1 (HMGA1) transcriptome in cancer and development. Curr. Mol. Med. 2016, 16, 353-393. [CrossRef]

19. Fusco, A.; Fedele, M. Roles of HMGA proteins in cancer. Nat. Rev. Cancer 2007, 7, 899-910. [CrossRef] [PubMed]

20. Fedele, M.; Fusco, A. HMGA and cancer. Biochim. Biophys. Acta 2010, 1799, 48-54. [CrossRef] [PubMed]

21. Tesfaye, A.; DiCello, F.; Hillion, J.; Ronnett, B.M.; Elbahloul, O.; Ashfaq, R.; Dhara, S.; Prochownik, E.; Tworkoski, K.; Reeves, R.; et al. The high-mobility group A1 gene up-regulates cyclooxygenase 2 expression in uterine tumorigenesis. Cancer Res. 2007, 67, 3998-4004. [CrossRef] 
22. Hillion, J.; Roy, S.; Heydarian, M.; Cope, L.; Xian, L.; Koo, M.; Luo, L.Z.; Kellyn, K.; Ronnett, B.M.; Huso, T. The High Mobility Group A1 (HMGA1) gene is highly overexpressed in human uterine serous carcinomas and carcinosarcomas and drives Matrix Metalloproteinase-2 (MMP-2) in a subset of tumors. Gynecol. Oncol. 2016, 141, 580-587. [CrossRef] [PubMed]

23. Wei, L.; Liu, X.; Zhang, W.; Wei, Y.; Li, Y.; Zhang, Q.; Dong, R.; Kwon, J.S.; Liu, Z.; Zheng, W.; et al. Overexpression and oncogenic function of HMGA2 in endometrial serous carcinogenesis. Am. J. Cancer Res. 2016, 6, 249-259.

24. Esposito, F.; De Martino, M.; Petti, M.G.; Forzati, F.; Tornincasa, M.; Federico, A.; Arra, C.; Pierantoni, G.M.; Fusco, A. HMGA1 pseudogenes as candidate proto-oncogenic competitive endogenous RNAs. Oncotarget 2014, 5, 8341-8354. [CrossRef] [PubMed]

25. Esposito, F.; De Martino, M.; D’Angelo, D.; Mussnich, P.; Raverot, G.; Jaffrain-Rea, M.L.; Fraggetta, F.; Trouillas, J.; Fusco, A. HMGA1-pseudogene expression is induced in human pituitary tumors. Cell Cycle 2015, 14, 1471-1475. [CrossRef]

26. Livak, K.J.; Schmittgen, T.D. Analysis of relative gene expression data using real-time quantitative PCR and the $2^{-\Delta \Delta C T}$ method. Methods 2001, 25, 402-408. [CrossRef]

27. Palumbo, J.A.; Meireles Da Costa, N.; Esposito, F.; De Martino, M.; D'Angelo, D.; de Sousa, V.P.; Martins, I.; Nasciutti, L.E.; Fusco, A.; Ribeiro Pinto, L.F. HMGA2 overexpression plays a critical role in the progression of esophageal squamous carcinoma. Oncotarget 2016, 7, 25872-25874. [CrossRef]

28. Palumbo, A., Jr.; De Martino, M.; Esposito, F.; Fraggetta, F.; Neto, P.N.; Valverde Fernandes, P.; Santos, I.C.; Dias, F.L.; Nasciutti, L.E.; Meireles Da Costa, N.; et al. HMGA2, but not HMGA1, are overexpressed in human larynx carcinomas. Histopathology 2018, 72, 1102-1114. [CrossRef]

29. R Core Team. R: A Language and Environment for Statistical Computing; R Foundation for Statistical Computing: Vienna, Austria, 2019.

30. Kessenbrock, K.; Plaks, V.; Werb, Z. Matrix metalloproteinases: Regulators of the tumor microenvironment. Cell 2012, 141, 52-67. [CrossRef]

31. Liau, S.S.; Jazag, A.; Whang, E.E. HMGA1 is a determinant of cellular invasiveness and in vivo metastatic potential in pancreatic adenocarcinoma. Cancer Res. 2006, 66, 11613-11622. [CrossRef]

32. Wang, J.; Zhao, X.; Guo, Z.; Ma, X.; Song, Y.; Guo, Y. Regulation of NEAT1/miR-214-3p on the growth, migration and invasion of endometrial carcinoma cells. Arch. Gynecol. Obstet. 2017, 295, 1469-1475. [CrossRef] [PubMed]

33. Abe, N.; Watanabe, T.; Sugiyama, M.; Uchimura, H.; Chiappetta, G.; Fusco, A.; Atomi, Y. Determination of high mobility group $\mathrm{I}(\mathrm{Y})$ expression level in colorectal neoplasias: A potential diagnostic marker. Cancer Res. 1999, 59, 1169-1174. [PubMed]

34. Chiappetta, G.; Manfioletti, G.; Pentimalli, F.; Abe, N.; Di Bonito, M.; Vento, M.T.; Giuliano, A.; Fedele, M.; Viglietto, G.; Santoro, M.; et al. High mobility group HMGI(Y) protein expression in human colorectal hyperplastic and neoplastic diseases. Int. J. Cancer 2001, 91, 147-151. [CrossRef]

35. Piscuoglio, S.; Zlobec, I.; Pallante, P.; Sepe, R.; Esposito, F.; Zimmermann, A.; Diamantis, I.; Terracciano, L.; Fusco, A.; Karamitopoulou, E. HMGA1 and HMGA2 protein expression correlates with advanced tumour grade and lymph node metastasis in pancreatic adenocarcinoma. Histopathology 2012, 60, 397-404. [CrossRef] [PubMed]

36. Zhang, Z.; Wang, Q.; Chen, F.; Liu, J. Elevated expression of HMGA1 correlates with the malignant status and prognosis of non-small cell lung cancer. Tumour Biol. 2015, 36, 1213-1219. [CrossRef] [PubMed]

37. Liau, S.S.; Rocha, F.; Matros, E.; Redston, M.; Whang, E. High mobility group AT-hook 1 (HMGA1) is an independent prognostic factor and novel therapeutic target in pancreatic adenocarcinoma. Cancer 2008, 113, 302-314. [CrossRef] [PubMed]

38. Chiappetta, G.; Botti, G.; Monaco, M.; Pasquinelli, R.; Pentimalli, F.; Di Bonito, M.; D’Aiuto, G.; Fedele, M.; Iuliano, R.; Palmieri, E.A.; et al. HMGA1 protein overexpression in human breast carcinomas: Correlation with ErbB2 expression. Clin. Cancer Res. 2004, 10, 7637-7644. [CrossRef]

39. Romero-Pérez, L.; Castilla, M.Á.; López-García, M.Á.; Díaz-Martín, J.; Biscuola, M.; Ramiro-Fuentes, S.; Oliva, E.; Matias-Guiu, X.; Prat, J.; Cano, A.; et al. Molecular events in endometrial carcinosarcomas and the role of high mobility group AT-hook 2 in endometrial carcinogenesis. Hum. Pathol. 2013, 44, 244-254. [CrossRef] 
40. Ma, J.; Li, D.; Kong, F.F.; Yang, D.; Yang, H.; Ma, X.X. miR-302a-5p/367-3p-HMGA2 axis regulates malignant processes during endometrial cancer development. J. Exp. Clin. Cancer Res. 2018, 37, 19. [CrossRef]

41. Montserrat, N.; Mozos, A.; Llobet, D.; Dolcet, X.; Pons, C.; de Herreros, A.G.; Matias-Guiu, X.; Prat, J. Epithelial to mesenchymal transition in early stage endometrioid endometrial carcinoma. Hum. Pathol. 2012, 43, 632-643. [CrossRef]

42. Li, Y.; Zhao, Z.; Xu, C.; Zhou, Z.; Zhu, Z.; You, T. HMGA2 induces transcription factor Slug expression to promote epithelial-to-mesenchymal transition and contributes to colon cancer progression. Cancer Lett. 2014, 355, 130-140. [CrossRef] [PubMed]

43. Palumbo Júnior, A.; Meireles Da Costa, N.; Esposito, F.; Fusco, A.; Ribeiro Pinto, L.F. High Mobility Group A proteins in esophageal carcinomas. Cell Cycle 2016, 15, 2410-2413. [CrossRef] [PubMed]

C 2019 by the authors. Licensee MDPI, Basel, Switzerland. This article is an open access article distributed under the terms and conditions of the Creative Commons Attribution (CC BY) license (http://creativecommons.org/licenses/by/4.0/). 